\title{
Nanoflow hydrodynamics
}

\author{
J. S. Hansen* and Jeppe C. Dyre \\ Danish National Research Foundation (DNRF) Centre “Glass and Time”, IMFUFA, Department of Science, Systems and Models, Roskilde \\ University, Postbox 260, DK-4000 Roskilde, Denmark \\ Peter J. Daivis ${ }^{\ddagger}$ \\ Applied Physics, School of Applied Sciences, RMIT University, GPO Box 2476, Melbourne, Victoria 3001, Australia \\ B. D. Todd ${ }^{\S}$ \\ Faculty of Engineering \& Industrial Sciences, Swinburne University of Technology, P.O. Box 218, Hawthorn, Victoria 3122, Australia
}

Henrik Bruus\|

Department of Micro- and Nanotechnology, Technical University of Denmark, DTU Nanotech Building 345 B, DK-2800 Kongens Lyngby, Denmark

(Received 28 March 2011; revised manuscript received 4 August 2011; published 14 September 2011)

\begin{abstract}
We show by nonequilibrium molecular dynamics simulations that the Navier-Stokes equation does not correctly describe water flow in a nanoscale geometry. It is argued that this failure reflects the fact that the coupling between the intrinsic rotational and translational degrees of freedom becomes important for nanoflows. The coupling is correctly accounted for by the extended Navier-Stokes equations that include the intrinsic angular momentum as an independent hydrodynamic degree of freedom.
\end{abstract}

DOI: 10.1103/PhysRevE.84.036311

PACS number(s): 47.61.-k, 47.11.Mn

\section{INTRODUCTION}

Flow of gases and liquids is described by the NavierStokes equation dating back almost 200 years [1,2]. For an incompressible fluid and in the absence of a pressure gradient the Navier-Stokes equation is $\rho D \mathbf{u} / D t=\rho \mathbf{F}_{e}+$ $\eta_{0} \nabla^{2} \mathbf{u}$, where $\rho$ is the density, $\mathbf{u}=\mathbf{u}(\mathbf{r}, t)$ the velocity field, $D / D t=\partial / \partial t+\mathbf{u} \cdot \boldsymbol{\nabla}$ is the local material time derivative, $\mathbf{F}_{e}$ the external force per unit mass, and $\eta_{0}$ the shear viscosity. The Navier-Stokes equation describes very well the fluid dynamics of a wide range of situations, and it is routinely used for investigating phenomena as diverse as the air flow around a wing, a tsunami, water flow in pipes and channels, etc. [3,4]. Even the intriguing turbulence phenomenon is captured by the Navier-Stokes equation, which has solutions that become unstable and chaotic at large flow velocities just as real flows do [5-7].

The Navier-Stokes equation is derived by combining momentum conservation with linear constitutive equations expressing proportionality between thermodynamical forces and corresponding fluxes [8]. For example, the shear stress (transverse momentum flux) is linearly dependent on the traceless symmetric part of the velocity gradient tensor. The flux of intrinsic angular momentum (or spin), as well as its coupling to the translational momentum, is usually ignored in the hydrodynamical description. Exactly what role does this play for the fluid dynamics? Of course, for flows of pointlike particles it does not introduce any new physics, because in this

\footnotetext{
*jschmidt@ruc.dk

$\dagger$ dyre@ruc.dk

${ }^{\ddagger}$ peter.daivis@rmit.edu.au

§btodd@swin.edu.au

"bruus@nanotech.dtu.dk
}

case the spin angular momentum is zero. For molecular fluids the spin angular velocity $\boldsymbol{\Omega}$, however, deviates from $(\boldsymbol{\nabla} \times \mathbf{u}) / 2$, giving rise to a thermodynamic force. This possibility leads to an extra channel for energy dissipation, thereby increasing the viscous dissipation $[8,9]$. Consider a steady shear flow along a wall where the molecules have nonzero spin in the bulk. As shown from molecular dynamics simulations [10] the spin angular velocity follows a no-slip boundary condition, which requires that the angular velocity is zero at the wall. Consequently, spin angular momentum is transported to the wall where it is absorbed, leading to increase of the energy dissipation.

Little attention has been given to the hydrodynamic angular velocity degree of freedom for the simple reason that it plays no role for flows on macroscopic length scales $[8,11]$. This paper shows, however, that a fluid-mechanical description of nanoflows of molecular liquids must include the spin angular velocity in order to be quantitatively accurate. We show this by considering results from nonequilibrium molecular dynamics simulations of a system of argon atoms, where the standard Navier-Stokes equation works well, and simulations of water, where this is not the case because the molecular spin is included in the description.

The extension of the Navier-Stokes equation to deal with the coupling between the hydrodynamic flow degree of freedom $\mathbf{u}(\mathbf{r}, t)$ and the microscopic molecular spin angular velocity degree of freedom $\boldsymbol{\Omega}(\mathbf{r}, t)$ was developed long ago [12-14]. If $I$ is $1 / 3$ of the trace of the inertia tensor per unit mass, in the absence of torque and pressure gradients the extended NavierStokes equations $[14,15]$ read for a divergence-free flow

$$
\begin{aligned}
\rho \frac{D \mathbf{u}}{D t} & =\rho \mathbf{F}_{e}+\left(\eta_{0}+\eta_{r}\right) \nabla^{2} \mathbf{u}+2 \eta_{r} \boldsymbol{\nabla} \times \boldsymbol{\Omega}, \\
\rho I \frac{D \boldsymbol{\Omega}}{D t} & =2 \eta_{r}(\boldsymbol{\nabla} \times \mathbf{u}-2 \boldsymbol{\Omega})+\zeta \nabla^{2} \boldsymbol{\Omega} .
\end{aligned}
$$


The transport coefficients $\eta_{r}$ and $\zeta$ are referred to as the rotational and spin viscosities. For a flow of pointlike particles $\boldsymbol{\Omega}=(\boldsymbol{\nabla} \times \mathbf{u}) / 2$; in this case, via the incompressible fluid identity $\nabla \times(\nabla \times \mathbf{u})=-\nabla^{2} \mathbf{u}$, the first equation reduces to the standard Navier-Stokes equation.

The coupling occurs because the pressure tensor for a molecular fluid may in general be a nonsymmetric quantity because the force vector acting between a pair of molecules is not parallel to the vector between their molecular centers of mass. If, on average, the vorticity is not equal to twice the molecular angular velocity, a thermodynamical force arises [14]. In the absence of spin diffusion, this thermodynamic force changes the angular velocity until the molecules rotate at a rate consistent with the local vorticity. This condition is denoted the local rigidity condition and the corresponding thermodynamic force is referred to as the sprain rate [14]. For the fluid to be locally rigid the vorticity (or local rotation) must be equal to $2 \boldsymbol{\Omega}$, i.e., the sprain rate is expressed as $\boldsymbol{\nabla} \times \mathbf{u}-2 \boldsymbol{\Omega}$. In Eq. (1b) we see this thermodynamic force entering as the first term on the right-hand side. The rotational viscosity thus describes the decay of the antisymmetric stress which is a local process and originates from molecular intrinsic angular momentum relaxation [11]. The diffusion of angular velocity is governed by a relation analogous to Newton's law of viscosity, i.e., the spin viscosity $\zeta$ relates the flux of angular momentum to the gradient in angular velocity (the thermodynamical force).

The first term on the right-hand side of Eq. (1b) can be regarded as a production term, i.e., this describes a nonconserved quantity. We note here that it is the total angular momentum which is conserved; Eq. (1b) only describes the intrinsic part. Finally, we note that in the presence of temperature gradients cross-coupling terms appear in the constitutive relations and an additional equation is needed to describe the dynamics of the energy [14].

To estimate the effect of the coupling one needs to know the values of the three transport coefficients, $\eta_{0}, \eta_{r}$, and $\zeta$. While data for the shear and rotational viscosities for water is available [16-18], data for the spin viscosity is very sparse. Bonthuis et al. [19] suggested an estimate of the spin viscosity through the rotational viscosity via $\zeta \approx a^{2} \eta_{r}$, leading to a spin viscosity of the order of $10^{-23} \mathrm{~kg} \mathrm{~m} \mathrm{~s}^{-1}$. Recently Hansen et al. [11] used equilibrium molecular dynamics (EMD) simulations to calculate $\eta_{0}, \eta_{r}$, and $\zeta$ for liquid water in the temperature range $T=284-319 \mathrm{~K}$. They found the spin viscosity to be in the order of $10^{-21} \mathrm{~kg} \mathrm{~m} \mathrm{~s}^{-1}$, i.e., two orders of magnitude larger than the estimate of Bonthuis et al. From the spin viscosity and the rotational viscosity one can define a characteristic length $l_{c}=\sqrt{\zeta / \eta_{r}}$ below which the angular momentum diffusion becomes important compared to the coupling to the fluid linear momentum. Using the value for the spin viscosity found in Ref. [11] means that $l_{c} \approx 3.4 \mathrm{~nm}$. Today nanochannels with a width down to $7 \mathrm{~nm}$ can be fabricated [20-22]; thus if the spin viscosity is in the order of $10^{-21} \mathrm{~kg} \mathrm{~m} \mathrm{~s}^{-1}$, we now approach the characteristic length scale $l_{c}$ and it is relevant to ask whether the effect of the coupling should be included in order to correctly account for the fluid dynamics. Using $\zeta \approx 10^{-23} \mathrm{~kg} \mathrm{~m} \mathrm{~s}^{-1}$, on the other hand, the characteristic length is approximately the size of a water molecule, predicting a negligible effect from the coupling.
For flow with low Reynolds number and where angular momentum diffusion is ignored, $\zeta=0$, one can see from Eq. (1b) that $\boldsymbol{\Omega}=(\boldsymbol{\nabla} \times \mathbf{u}) / 2$. Substituting this into Eq. (1a), one arrives at the classical Navier-Stokes formulation, i.e., the coupling has no effect on the flow profile. This is in agreement with the definition of the characteristic length $l_{c}$ and the discussion above: Simply ignoring the angular momentum diffusion, the critical length is $l_{c}=0$, meaning that there exists no effect from the coupling. By comparing the solution to Eqs. (1) with the classical solution for a Poiseuille flow, it can easily be shown that the flow rate reduction increases for decreasing channel width [11], indicating that the viscous dissipation due to the angular momentum diffusion becomes important on the nanoscale, but can be ignored on the macroscopic scale.

It has been shown that for simple atomic fluids confined to length scales of $\sim 3 \mathrm{~nm}$ the classical Navier-Stokes equation can be applied to predict the correct velocity profile $[23,24]$. However, it remains to be investigated whether the extended Navier-Stokes equations are valid on such small length scales, i.e., whether the continuum description is able to describe correctly the dynamics of highly confined fluids composed of nonspherical molecules.

In this paper we present results from extensive nonequilibrium molecular dynamics (NEMD) simulations of a planar Poiseuille flow of water confined in nanoslit pores with width of 3.6-6.2 nm. We compare the streaming velocity profile from the NEMD simulations with predictions from the extended Navier-Stokes equations using the transport coefficients found in Ref. [11], i.e., calculated from independent simulations using the EMD technique. The comparison thereby investigates (i) whether the extended Navier-Stokes equations apply on very small length scales, (ii) whether the value for the spin viscosity obtained in Ref. [11] is correct, and finally (iii) the effect from the coupling between the spin angular momentum of the molecules and the fluid translational momentum.

\section{MOLECULAR DYNAMICS}

In Ref. [11] we used the flexible simple point-charge water model $(\mathrm{SPC} / \mathrm{Fw})[16]$, where the electrostatic interactions between the atoms in different molecules are evaluated via the modified short-ranged Wolf method $[25,26]$. To enable a direct comparison we must therefore adopt this model and we will denote it as the SPC/Fw (Wolf) model. For more details about this particular water model and its parametrization we refer the reader to Refs. [16] and [11]. The software is written in-house and has been tested and extended for many years.

The two confining walls are composed of simple LennardJones particles [27]. The wall particles interact with other wall particles and the oxygen atoms in the water using the truncated and shifted 12-6 Lennard-Jones potential with parameters corresponding to oxygen-oxygen interactions, i.e., $m=16 \mathrm{~g} \mathrm{~mol}^{-1}, \sigma=3.19 \AA$, and $\epsilon / k_{B}=78.197 \mathrm{~K}$, such that wall-wall and fluid-wall Lennard-Jones interactions are the same. The cutoff is set to $2.5 \sigma$. The particles are arranged on a body-centered-cubic (bcc) lattice with a number density of $0.9 \sigma^{-3}$ and cannot be penetrated by the water. Each 


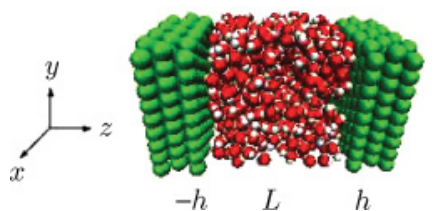

FIG. 1. (Color online) Snapshot from the simulation just after the wall particles (online: green/print: outer spheres) have been added. The wall particle diameter is the same as the oxygen (online: red/print: inner spheres) atomic diameter, but has been displayed differently for clarity. $L$ denotes the width of the pore and $h=L / 2$ the half width.

wall consists of three layers (see Fig. 1), giving a total of between 480 and 726 wall particles dependent on of the equilibration procedure (see below). The wall is designed to be weakly hydrophilic by letting the wall particles have alternately charges $\pm e / 4$, where $e$ is the elementary charge. The electrostatic force between the wall particles and water atoms are also evaluated using the modified Wolf method $[25,26]$. We note that the net charge is zero in each layer of the wall and that there will not be any layering effect due to the electrostatic interactions between the wall and fluid. Beside the Lennard-Jones and the electrostatic interactions with the water, the wall particles are held fixed around their lattice sites using a restoring spring force with a spring constant $k_{s}=1.6 \mathrm{~kg} \mathrm{~s}^{-2}$ [24] and were coupled to a Nosé-Hoover thermostat $[28,29]$ to keep the wall temperature fixed at approximately $T=312 \mathrm{~K}$.

Initially, the water molecules are equilibrated without the presence of the walls using a standard EMD simulation. This equilibration involves an isotropic compression of a rectangular simulation box with the $z$ direction being the longest direction and the $x$ and $y$ directions the shortest. This compression is done until a desired density is obtained, which means that the length in all directions will vary and therefore also the number of wall atoms. After equilibration the walls are placed on each side of the system, such that the direction of confinement is in the $z$ direction and the overall density is $\rho \approx 998 \mathrm{~kg} \mathrm{~m}^{-3}$. We then allow for further equilibration of the intramolecular degrees of freedom. After this second equilibration the external force field is applied to each atom in the $x$ direction $\rho \mathbf{F}_{e}=\rho\left(F_{e}, 0,0\right)$. Thus, the result is similar to a pressure-driven Poiseuille flow with a constant pressure gradient. Periodic boundary conditions are applied in all directions.

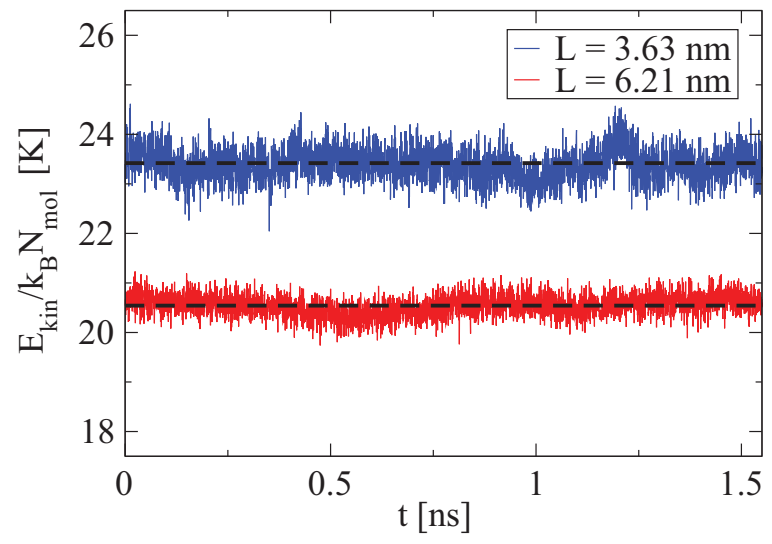

FIG. 2. (Color online) Kinetic energy as a function of time for two different channel widths $L=3.63 \mathrm{~nm}$ (upper curve, online: blue) and $6.31 \mathrm{~nm}$ (lower curve, online: red). The dashed lines indicate the overall mean kinetic energy.

The equations of motion for all particles are integrated using a leapfrog algorithm [30]. Each NEMD simulation cover a time span of $\sim 16 \mathrm{~ns}$ using a time step of $0.77 \mathrm{fs}$. For each channel width the molecular dynamics data are ensemble averaged over ten independent runs enabling an estimate the statistical error. After every 20 time steps the velocity, kinetic temperature, and density profiles are sampled using standard binning methods giving a total of $\sim 10 \times 10^{6}$ samples per system. To check the convergence, the simulation time is doubled for one of the simulations for each channel width and the velocity profile compared with the other shorter simulations. Furthermore, during the simulation runs the total kinetic energy is also monitored showing that the system has reached a steady state (see Fig. 2).We note that applying an external force field results in frictional heating which is conducted away at the wall-fluid boundary; however, the resulting temperature may vary. In Table I we list the kinetic temperature for each system as well as the corresponding values of the extrapolated transport coefficients.

It is worth noticing that the weak Coulomb interactions between the wall and fluid affect the bulk properties of the water. In Fig. 3(a) we have plotted the density profile in the situation where the wall particles are charged (hydrophilic) and where the wall is uncharged (hydrophobic) and where the field is turned off. It is seen that the bulk density is larger in the hydrophobic situation, which is, of course, directly linked to

TABLE I. Simulation system details and corresponding parameter values for the SPC/Fw (Wolf) model used for the comparison. The transport coefficients are extrapolated from Ref. [11].

\begin{tabular}{|c|c|c|c|c|c|c|}
\hline$N_{\text {water }}$ & $\begin{array}{c}L \\
(\mathrm{~nm})\end{array}$ & $\begin{array}{c}F_{e} \\
\left(\mathrm{~m} \mathrm{~s}^{-2}\right)\end{array}$ & $\begin{array}{c}T \\
(\mathrm{~K})\end{array}$ & $\begin{array}{c}\eta_{0} \\
\left(10^{-4} \mathrm{~Pa} \mathrm{~s}\right)\end{array}$ & $\begin{array}{c}\eta_{r} \\
\left(10^{-4} \mathrm{~Pa} \mathrm{~s}\right)\end{array}$ & $\begin{array}{c}\zeta \\
\left(10^{-21} \mathrm{~kg} \mathrm{~m} / \mathrm{s}\right)\end{array}$ \\
\hline 750 & 3.57 & 30.2 & 328.4 & $3.2 \pm 0.1$ & 1.678 & $1.01 \pm 0.04$ \\
\hline 825 & 3.63 & 30.2 & 335.0 & $2.5 \pm 0.9$ & 1.678 & $0.8 \pm 0.03$ \\
\hline 1050 & 3.92 & 10.0 & 331.7 & $2.9 \pm 0.2$ & 1.678 & $0.93 \pm 0.03$ \\
\hline 1125 & 4.18 & 5.0 & 321.4 & $4.1 \pm 0.7$ & 1.678 & $1.31 \pm 0.05$ \\
\hline 1200 & 4.59 & 4.0 & 327.6 & $3.3 \pm 0.6$ & 1.678 & $1.07 \pm 0.02$ \\
\hline 1275 & 5.03 & 4.0 & 312.8 & $5.2 \pm 0.1$ & 1.678 & $1.65 \pm 0.04$ \\
\hline 1500 & 6.21 & 2.0 & 316.4 & $4.8 \pm 0.1$ & 1.678 & $1.52 \pm 0.07$ \\
\hline
\end{tabular}



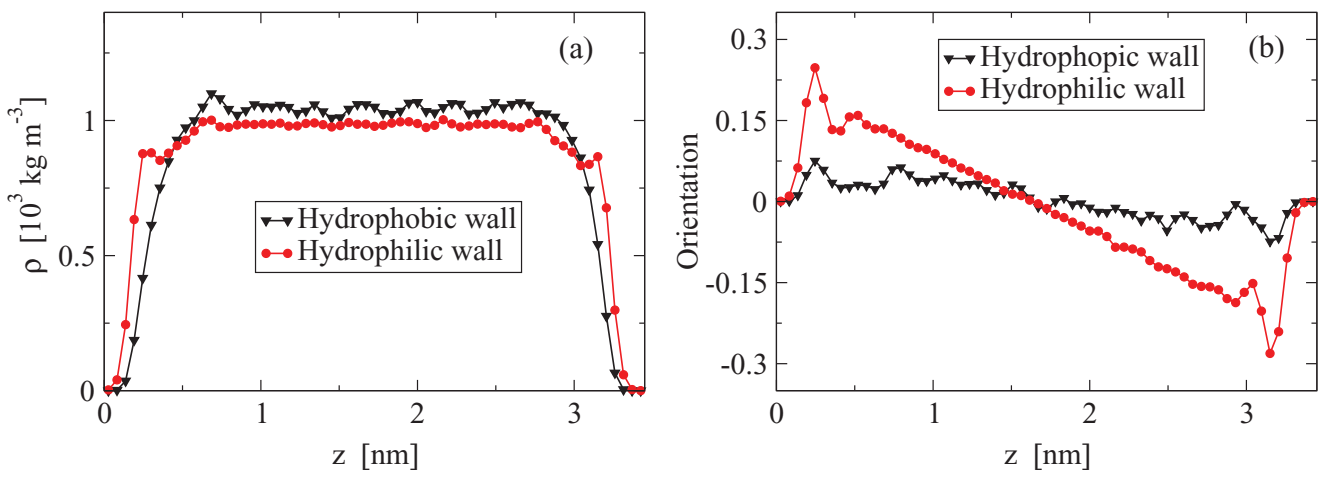

FIG. 3. (Color online) (a) Center-of-mass density profile in the cases of hydrophobic and hydrophilic walls. Channel width is $3.57 \mathrm{~nm}$. (b) The corresponding orientational parameter profile. In both (a) and (b) the results are from one simulation with the field turned off.

the large contact angle between the water and the uncharged wall. This also explains why flow of water confined between two hydrophobic walls lead to a plug flow characterized by a flat velocity profile and very different dynamics. It is important to note, however, that the hydrophilic walls do not introduce any long-ranged layering of the water molecules inside the channel and one can therefore expect the fluid to exhibit bulk behavior just a few molecular diameters from the wall. This is consistent with previous molecular dynamics results by Priezjev [31] of atomic fluids where the wall-fluid interaction strength was varied which affected the slip length, but not the bulk fluid properties.

In Fig. 3(b) we have plotted the orientation parameter profile $\theta_{z}$, defined as $\theta_{z}=\arccos \left(p_{z} /|\mathbf{p}|\right)-\pi / 2$, where $p_{z}$ is the $z$ component of the total dipole moment $\mathbf{p}$ of the water molecules in a slab. Thus, for a perfect uniform distribution of angles the orientation parameter is zero, i.e., this corresponds to the situation where no alignment of the water molecules takes place. Of course, the dipole moment can also be perfectly aligned with the plane parallel to the wall given $\theta_{z}=0$ : Using the $x$ and $y$ components of the dipole moment vector produces the same result as Fig. 3(b). From this we see that the hydrophilic wall-fluid interactions introduce an additional orientation that corresponds to a deviation in the order of $\sim 5 \%$ away from a perfectly uniform orientation. The relatively small increased orientation for a zero field system is likely linked to the Wolf method applied [32,33] and is removed when the field is switched on. It is important to point out that we have applied the Wolf method in order to enable a direct comparison between the NEMD simulations and the continuum prediction based on the transport coefficients in Ref. [11] where this method was applied.

To illustrate the effect of the coupling further we also perform a NEMD simulation series of a Poiseuille flow of argon where the coupling is absent. These simulations where carried out at the state point $(\rho, T)=\left(1247.4 \mathrm{~kg} \mathrm{~m}^{-3}, 239.6 \mathrm{~K}\right)$, which corresponds to a supercritical fluid.

\section{RESULTS}

For the geometry shown in Fig. 1 the extended NavierStokes equations can be solved analytically with Dirichlet slip boundary conditions where $u_{x}( \pm h)=u_{\text {slip }}$ and $\Omega_{y}( \pm h)=0$, giving [34]

$$
\begin{aligned}
u_{x}(z)= & u_{c}\left[1-z^{2}+\frac{2 \eta_{r} \operatorname{coth}(K h)}{\left(\eta_{r}+\eta_{0}\right) K h}\left(\frac{\cosh (K h z)}{\cosh (K h)}-1\right)\right] \\
& +u_{\text {slip }}
\end{aligned}
$$

Here $u_{x}$ is the $x$ component of the velocity field, $u_{c}=$ $h^{2} \rho F_{e} /\left(2 \eta_{0}\right), K=\left\{4 \eta_{r} \eta_{0} /\left[\zeta\left(\eta_{r}+\eta_{0}\right)\right]\right\}^{\frac{1}{2}}$, and $-1 \leqslant z \leqslant 1$ such that $u_{x}(1)=u_{x}(-1)=u_{\text {slip }}$. Ignoring the internal degrees of freedom this reduces to the solution of the classical planar Poiseuille flow

$$
u_{x}(z)=u_{c}\left(1-z^{2}\right)+u_{\text {slip }}
$$

If we know the values of all relevant transport coefficients [11], it is possible to make a direct comparison between the continuum Navier-Stokes predictions and data from the NEMD simulations once the slip velocity $u_{\text {slip }}$ is found.

It is well known that in the wall-fluid boundary region, the fluid is characterized by large density variations $[35,36]$ and anisotropy [15]. In this region the tensorial nonlocal transport coefficients should be included in a correct hydrodynamical description [8,37,38]. Thus, we cannot expect Eqs. (2) and (3) to hold very close to the wall-fluid boundary, but only in the interior of the channel where the fluid has bulk transport properties. This implies that when the analytical solutions of the Navier-Stokes and the extended Navier-Stokes equations are compared to data, only interior points should be considered. To find the slip velocity we use two different procedures. (i) A second-order polynomial was fitted to the interior NEMD data points and the slip velocity was found by extrapolating this polynomial to $\pm h$. (ii) The solutions to the classical and extended Navier-Stokes equations were fitted using the least-squares method to the interior NEMD data using only $u_{\text {slip }}$ as a fitting parameter. We let interior points be points that are more than $0.5 \mathrm{~nm}$ away from the walls. We further note that when the slip velocity is found by extrapolation it corresponds to the apparent slip velocity and that the slip velocity is the only fitting parameter used when we compare the velocity profiles.

Figure 4(a) shows the predicted velocity profile together with NEMD data for fluid argon confined in a slit pore of width $4.05 \mathrm{~nm}$ corresponding to approximately 11 atomic diameters. Here $u_{\text {slip }}$ is found from method (i) described above. The interior part of the channel is indicated by the two vertical dashed 

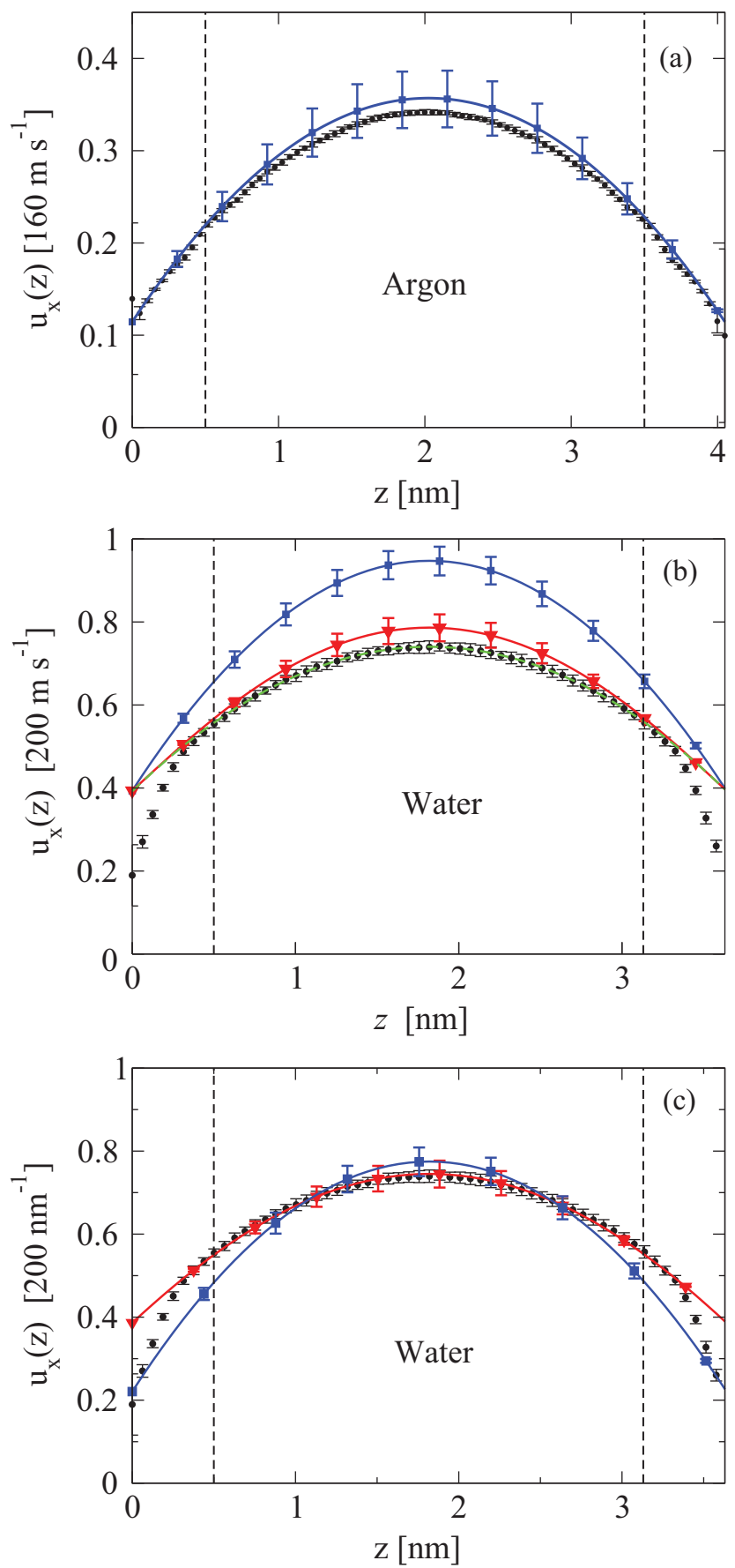

FIG. 4. (Color online) (a) Streaming velocity profile for argon fluid in a nanoslit pore of width $4.05 \mathrm{~nm}$ (11 atomic diameters). Line connected with filled squares represents the predicted profile, Eq. (3), using $\eta_{0}=1.5 \pm 0.2 \times 10^{-4} \mathrm{~Pa}$ s [40], and black filled circles represent the NEMD data. Standard deviations on the predicted profile are due to statistical uncertainties in the viscosity estimates. (b) Velocity profile for water in a nanoslit pore of width $3.63 \mathrm{~nm}(11$ molecular diameters). Line connected with filled squares represents the predicted profile using the classical Navier-Stokes theory, Eq. (3), line connected with triangles the extended Navier-Stokes theory, Eq. (2), and filled circles the NEMD data. $u_{\text {slip }}$ is found from fitting a second-order polynomial (dashed line) to data and extrapolating to $z=0 \mathrm{~nm}$. (c) As in (b), but where Eqs. (2) and (3) are fitted directly to interior NEMD data points. For all figures the two vertical dashed lines limit the interior part of the channel. lines. The classical theory is clearly capable of predicting the result from the simulation. The result is interesting on its own, since it indicates that the classical continuum fluid dynamical description is applicable on extremely small length scales for atomic structureless fluids. This has also been shown previously-see, for example, Refs. [23,24]. It is worth mentioning that this surprising observation is also supported by the fact that the wave-vector-dependent viscosity is constant with a value of the macroscopic viscosity for wavelengths up to $0.25 \AA^{-1}$ for liquid argon-see also Ref. [39]. In Fig. 4(b) we plot the corresponding system for water, where the channel is again 11 molecular diameters wide. It is clearly seen that the classical Navier-Stokes theory, which excludes the coupling phenomenon, fails to predict the correct velocity profile, whereas the extended theory agrees well with the NEMD data in the interior part of the pore. The values of the transport coefficients used in Figs. 4(b) and 4(c) are listed in Table I. One could argue that the classical Navier-Stokes theory fails due an incorrect slip velocity estimate. Figure 4(c) shows least-squares fit of the solutions to the classical and extended Navier-Stokes equations to the interior NEMD data points. We stress that only the slip velocity is used as a fitting parameter according to method (ii) above. Again, the extended theory agrees with the NEMD data in the interior part of the channel, whereas the classical theory fails.

If one evaluates the profile curvature in the channel midpoint, it is possible to compare the molecular dynamics description with the continuum predictions directly without having to perform any fitting. To this end we note that the predicted curvatures of the continuum theory can be found via the second-order derivative of Eqs. (2) and (3). The relative difference between the classical and extended predictions is then given by

$$
C_{\mathrm{rel}}=1-\frac{\eta_{r} \operatorname{coth}(K h) K h}{\left(\eta_{r}+\eta_{0}\right) \cosh (K h)},
$$

such that, if $C_{\text {rel }}=1$, there is no effect from the coupling on the velocity profile curvature. In Fig. 5 we compare $C_{\text {rel }}$ for the different pore widths as predicted by Eq. (4) with the NEMD data. It is seen that within statistical uncertainty there is good agreement between the NEMD data (circles) and

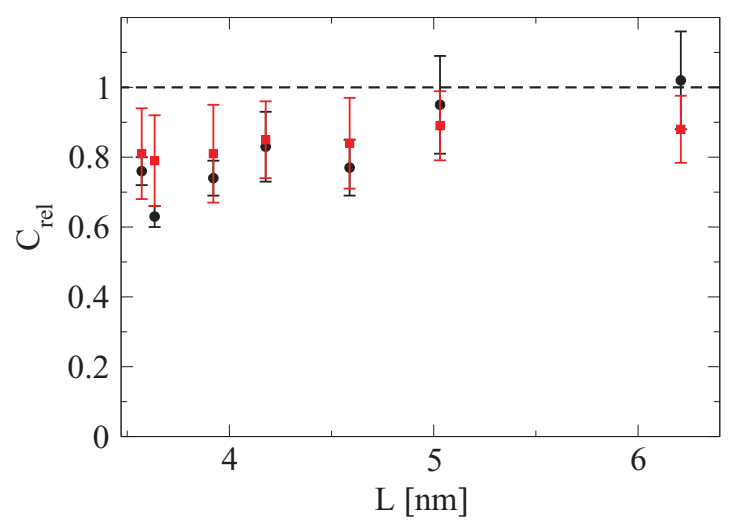

FIG. 5. (Color online) Relative difference in velocity profile curvature as a function of pore width. Filled circles represent NEMD data and squares the prediction from Eq. (4). The dashed line indicates $C_{\text {rel }}=1$ corresponding to the classical Navier-Stokes theory. 
the extended theory (squares). Moreover, as the pore width increases, the effect of the coupling vanishes (statistically), which is as expected.

\section{CONCLUSION}

The excellent agreement between the NEMD data and the extended Navier-Stokes equations leads us to conclude that the coupling between the spin angular momentum and the linear momentum is correctly accounted for by the extended continuum description. Furthermore, the comparison confirms the transport coefficients reported in Ref. [11]. These findings show that there is a significant coupling between the spin angular momentum and the linear momentum at very small confining geometries. From an application point of view this an important result, since the flow rate is significantly affected. Thus, in future development of nanofluidic devices where water flows in carbon nanotubes, for example, one must include the coupling effect in order to make the correct fluid dynamics predictions. Finally, we note that our simulations support the recent suggestion that one may drive a nanoflow of a dipolar fluid by a rotating electrical field [11,19].

\section{ACKNOWLEDGMENTS}

J.S.H. wishes to acknowledge Lundbeckfonden for supporting this work as part Grant No. R49-A5634. The center for viscous liquid dynamics "Glass and Time" is sponsored by the Danish National Research Foundation (DNRF).
[1] C. L. M. H. Navier, Mem. Acad. R. Sci. Inst. France 6, 389 (1823).

[2] G. G. Stokes, Mathematical and Physical Papers. Volume 1 (Oxford University Press, Oxford, UK, 1880).

[3] D. J. Tritton, Physical Fluid Dynamics (Oxford University Press, Oxford, UK, 1988).

[4] B. Lautrup, Physics of Continuous Matter (Institute of Physics, Bristol, UK, 2005).

[5] A. N. Kolmogorov, Dokl. Akad. Nauk SSSR 32, 16 (1941).

[6] S. B. Pope, Turbulent Flows (Cambridge University Press, Cambridge, UK, 2000).

[7] S. Grossmann, Rev. Mod. Phys. 72, 603 (2000).

[8] S. R. de Groot and P. Mazur, Nonequilibrium Thermodynamics (Dover, New York, 1984).

[9] J. S. Hansen, P. J. Daivis, and B. D. Todd, Microfluid. Nanofluid. 6, 785 (2009).

[10] K. P. Travis, B. D. Todd, and D. J. Evans, Physica A 240, 315 (1997).

[11] J. S. Hansen, H. Bruus, B. D. Todd, and P. J. Daivis, J. Chem. Phys. 133, 144906 (2010).

[12] M. Born, Z. Phys. 1, 221 (1920).

[13] N. K. Ailawadi, B. J. Berne, and D. Forster, Phys. Rev. A 3, 1462 (1971).

[14] D. J. Evans and W. B. Streett, Mol. Phys. 36, 161 (1978).

[15] J. Delhommelle and D. J. Evans, Mol. Phys. 100, 2857 (2002).

[16] Y. Wu, H. L. Tepper, and G. A. Voth, J. Chem. Phys. 124, 024503 (2006).

[17] CRC Handbook of Chemistry and Physics, edited by D. R. Lide (CRC, Cleveland, OH, 1976).

[18] D. Bertolini and A. Tani, Phys. Rev. E 52, 1699 (1995).

[19] J. D. Bonthuis, D. Horinek, L. Bocquet, and R. R. Netz, Phys. Rev. Lett. 103, 144503 (2009).

[20] F. Martin, R. Walczak, A. Boiarski, M. Cohen, T. West, C. Cosentino, and M. Ferrari, J. Controlled Release 102, 123 (2005).
[21] N. R. Tas, J. Haneveld, H. V. Jansen, M. Elwenspoek, and A. van den Berg, Appl. Phys. Lett. 85, 3274 (2004).

[22] L. H. Thamdrup, F. Persson, H. Bruus, H. K. Flyvbjerg, and A. Kristensen, Appl. Phys. Lett. 91, 163505 (2007).

[23] J. Koplik, J. R. Banavar, and J. F. Willemsen, Phys. Fluid. A 1, 781 (1989).

[24] K. P. Travis, B. D. Todd, and D. J. Evans, Phys. Rev. E 55, 4288 (1997).

[25] D. Wolf, P. Keblinski, S. R. Phillpot, and J. Eggebrecht, J. Chem. Phys. 110, 8254 (1999).

[26] D. Zahn, B. Schilling, and S. M. Kast, J. Phys. Chem. B 106, 10725 (2002).

[27] D. A. Mcquarrie, Statistical Mechanics (Harper and Row, New York, 1976).

[28] S. Nosé, Mol. Phys. 52, 255 (1984).

[29] W. G. Hoover, Phys. Rev. A 31, 1695 (1985).

[30] D. Frenkel and B. Smit, Understanding Molecular Simulation (Academic, London, 1996).

[31] N. V. Priezjev, Phys. Rev. E 75, 051605 (2007).

[32] S. E. Feller, R. W. Pastor, A. Rojnuckarin, S. Bogusz, and B. R. Brooks, J. Phys. Chem. 100, 17011 (1996).

[33] J. M. Rodgers and J. D. Weeks, Proc. Natl. Acad. Sci. USA 105, 19136 (2008).

[34] A. C. Eringen, Contribution to Mechanics, edited by D. Abir (Pergamon, Oxford, UK, 1969).

[35] J. P. Hansen and I. R. McDonald, Theory of Simple Liquids (Academic, Amsterdam, 2006).

[36] S. Toxvaerd, J. Chem. Phys. 74, 1998 (1981).

[37] I. Bitsanis, T. K. Vanderlick, M. Tirrell, and H. T. Davis, J. Chem. Phys. 89, 3152 (1988).

[38] B. D. Todd, J. S. Hansen, and P. J. Daivis, Phys. Rev. Lett. 100, 195901 (2008).

[39] J. S. Hansen, P. J. Daivis, K. P. Travis, and B. D. Todd, Phys. Rev. E 76, 041121 (2007).

[40] R. L. Rowley and M. M. Painter, Int. J. Therm. Phys. 18, 1109 (1997). 